\title{
Physical Model Investigation of a Compact Waste Water Pumping Station
}

\author{
Kilian Kirst ${ }^{1}$, D.-H. Hellmann ${ }^{1}$, Bernd Kothe $^{2}$ and Peer Springer ${ }^{2}$ \\ ${ }^{1}$ Department of Mechanical Engineering, Technical University Kaiserslautern \\ Gottlieb-Daimler-Straße, Kaiserslautern, D-67663, Germany \\ kkirst@rhrk.uni-kl.de, hellmann@mv.uni-kl.de \\ ${ }^{2}$ KSB AG, Johann-Klein-Straße 9, Frankenthal, D-67227, Germany \\ bernd.kothe@ksb.com, peer.springer@ksb.com
}

\begin{abstract}
To provide required flow rates of cooling or circulating water properly, approach flow conditions of vertical pump systems should be in compliance with state of the art acceptance criteria. The direct inflow should be vortex free, with low pre-rotation and symmetric velocity distribution. Physical model investigations are common practice and the best tool of prediction to evaluate, to optimize and to document flow conditions inside intake structures for vertical pumping systems. Optimization steps should be accomplished with respect to installation costs and complexity on site. The report shows evaluation of various approach flow conditions inside a compact waste water pumping station. The focus is on the occurrence of free surface vortices and the evaluation of air entrainment for various water level and flow rates. The presentation of the results includes the description of the investigated intake structure, occurring flow problems and final recommendations.
\end{abstract}

Keywords: physical model investigation, waste water, pumping station

\section{Introduction}

Together with the operating conditions the geometric parameters of an intake structure determine the approach flow conditions. The demand for small structures by increasing flow rates leads to conceptions of intake structures that sometimes outbid the recommendations of the common standards. In some cases limits specified in state of the art guidelines are exceeded, to reduce the volume of the intake to a minimum.

The capability of providing the required flow rates should always be proved by physical model investigations. Although numerical analysis are more and more adopted in this work field, they cannot be used in general. Especially the display and optimization of free surface problems, such as the occurrence of free surface vortex systems or air entrainment, are more accurately and efficiently performed with model tests. Physical model investigations for waste water pumping stations differ in some points to tests for cooling water systems. Normally water levels inside pumping stations are determinate by the water level of the connected open water (e.g., sea, river) outside the structure. The foundation depth of the pump sump must be adequate to adjust sufficient submergence of the pump impeller for low water levels to avoid NPSH problems.

Compared to cooling water pumping stations most types of sewage water pumping stations normally are charged at intervals. Problems arise when sedimentation and floating sludge occur. This leads to a different view on test procedures. The measurement of velocity distributions and pre-rotation at impeller elevation is similar to investigations for cooling water systems. Also the detection of possible vortex formations for all operating points is required. For some sewage pumping stations a maximum drainage level with sufficient operating conditions is specified. These shut-down thresholds can be determined in a model test by drop level tests. Therefore the water level is decreased until a vortex system not in compliance with state of the art specifications occurs.

Due to a large percentage of particulate material in sewage water, sedimentation can become a problem when low velocity areas develop inside the structure. Large clusters of solid matter can shorten the maintenance time or lead to a damage of the pumping system. Another main difference to cooling water is the occurrence of floating sludge. In prototype structures floating sludge can growth to a solid and thick layer, swimming on the water surface. To discharge the sludge out of the pump sump, the water level has to be decreased until it can be sucked into the inlet nozzle. If this is not possible, the sludge has to be removed mechanically.

Accepted for publication November 16 2010: Paper number O10029S

Corresponding author: Kilian Kirst, kkirst@rhrk.uni-kl.de

This paper was presented at 2010 International Conference on Pumps and Fans, October 18-21, 2010, Hangzhou, China 
The described problems can be avoided during the planning stage. In physical model investigations low velocity areas can be detected by injecting dye in the flow and by measuring flow distributions at critical regions. By installing flow guiding components, the flow at ground level as well as on the surface can be accelerated. In this way, an optimized distribution of the particulate material is achieved.

\section{Design Criteria for water intake structures}

Approach flow conditions of vertical pumping systems are determined by operating conditions and geometric parameters. The worldwide demand for smaller structures with increasing flow rates leads to conceptions of intake structures that outbid the recommendations of common standards. In some cases, limits specified in leading guidelines are exceeded, to reduce the volume of the intake to a minimum.

In all cases, the capability of providing the required flow rates probably should be proved by physical model investigations.

The basic design of an intake structure can be described by the following parameters:

- submergence $(\mathrm{S})$

- back wall (X) and floor clearance (C)

- bay width (W)

- length of approach flow channel (LD)

Typically, these parameters are related to the pump bell diameter $\mathrm{D}$.
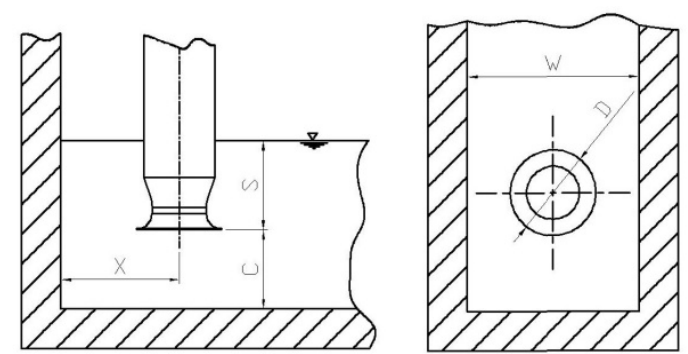

Fig. 1 Characteristic dimensions of a pump sump

The Hydraulic Institute Standard HIS and the reports of the British Hydraulic Research Association BHRA (published by Prosser) and Padmanabhan are the most common standards concerning physical model investigations. All geometrical dimensions of pump sumps can be specified by dimensionless figures (C/D, X/D, W/D and SD/D). [1][2]

\section{Physical model investigation}

With physical model investigations the approach flow conditions of intake structures can be evaluated, optimized and documented. The evaluation considers three main criteria:

- vortex formation

- pre-rotation

- velocity distribution

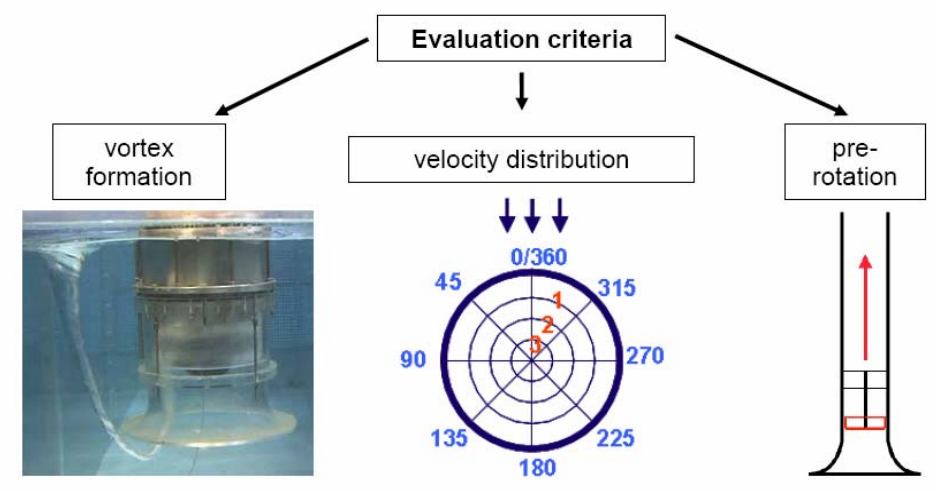

Fig. 2 Characteristic evaluation criteria for physical model investigations 
Geometrical and physical similarity are basic principles to allow a definite transfer of model test results to a prototype structure. By this means, preconditions have to be fulfilled to achieve similar flow conditions in the model and the prototype structure.

While geometric parameters are converted with the same scale factor, hydraulic parameters are quantified by dimensionless values. The most significant numbers determine the flow behavior for different basic conditions.

\section{Froude number: $\quad F r^{n}=\mathbf{c} \xi \sqrt{\mathbf{g D}}$}

The Froude number is the ratio of inertia and gravitational forces.

Weber number: $\quad W_{e}=\sqrt{\rho / / g}$

The Weber number describes flow conditions under surface stress effects.

Reynolds number: $\quad R \boldsymbol{s}=\boldsymbol{s d} / \mathbf{v}$

The Reynolds number gives a measure of the ratio of inertial forces to viscous forces. Froude number and Reynolds number are the most significant similarity figures concerning physical model investigations. Flow mainly influenced by friction, such as flow through closed pipes or covered sections, is characterized by the Reynolds number. Froude number must be equal for model and prototype structure, if gravity is the dominating force of the main flow. This involves all free surface flow.

Due to physical reasons, it is not possible to have equal Reynolds and Froude number at the same time, except for the trivial case of modeling in scale 1:1. Almost all intake structures are of free surface type. Thus the Froude law $\left(\mathrm{F}_{\text {Model }}=\mathrm{F}_{\text {Prototype }}\right)$ has to be applied, while the Reynolds number cannot be neglected $\left(\mathrm{Re}>\mathrm{Re}_{\text {crit }}\right)$.

Vortex formations are classified by different energy levels. With increasing strength, the vortex core emerges more defined. George Hecker from the Alden Research Laboratory (ARL) /2/ sections vortex formations into two groups: free surface and sub-surface boundary-attached vortices. Vortex classification is shown slightly modified in the following figures.

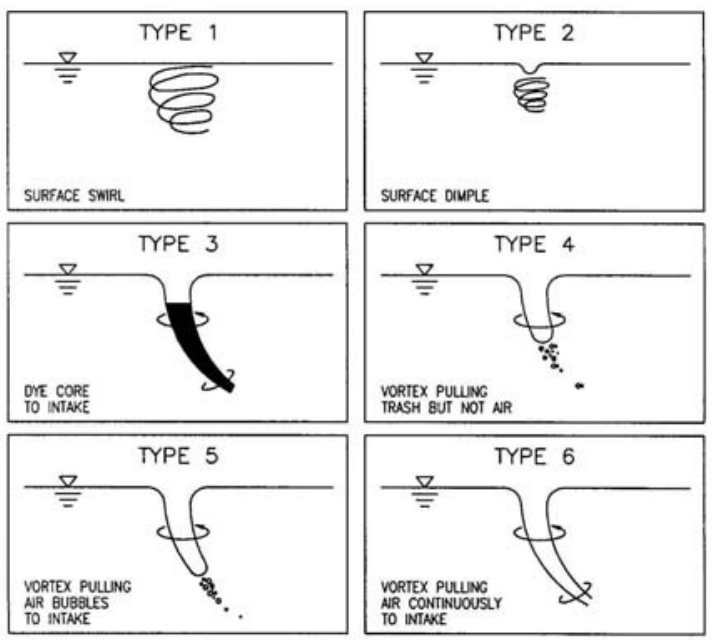

Fig. 3 Classification of free-surface vortices

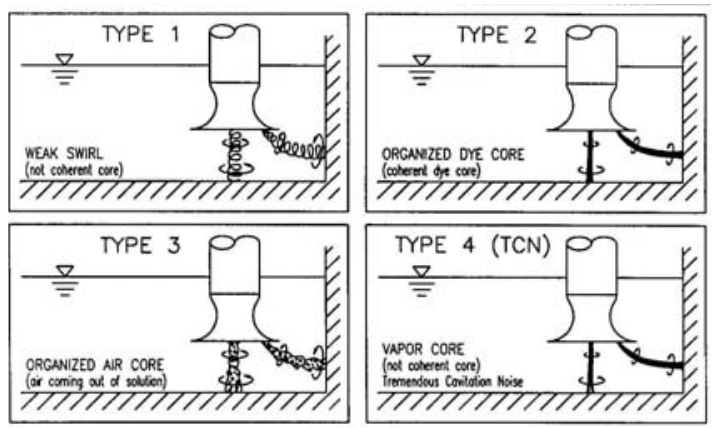

Fig. 4 Classification of sub-surface boundary attached vortices

According state of the art criteria, only free surface vortices of type 1 and 2 are accepted. Boundary-attached subsurface vortices are classified into four types, although the ARL distinguishes between three types. Type 4 (TCN-type) had been detected at TU Kaiserslautern and classified and appended to the excising Hecker scale.[3] According state of the art criteria, no boundary- 
attached subsurface vortex is accepted. [2]

As the focus of this work is on occurring vortex systems and air entrainment, the measurement procedures of the velocity distribution and the pre-rotation will not be described in detail.

All model investigations described in this paper were accomplished at a wet pit test rig. Model structures were made of Plexiglas, plastics and steel.

\section{Test and results}

The investigated waste water pumping station consists of a rectangular building, charged laterally by an inflow pipe. Three submersible motor pumps are positioned on a base at the top end of the building. $90^{\circ}$-floor splitters were installed under each inlet nozzle to improve the inflow conditions and to avoid floor attached sub-surface vortex system. A stilling chamber deflects the inflow with $90^{\circ}$ towards the pump sump. It is designed as a balcony with openings at ground level. The number and the alignment of the openings correspondents with the installed pumps. Guiding plates underneath, one at each opening, deflect the leaving flow towards the pumps.

Most of the dimensions, such as the volume of the stilling chamber and width of the structure, are reduced to a minimum. The aim of the investigation was to evaluate the flow behavior inside the minimized structure in regard to air entrainment, vortex formations, minimum water level and potential sedimentation areas. Therefore various tests with different flow rates and varying water level were carried out for different combinations of operating pumps.

The following figures show the modeled structure with its components.

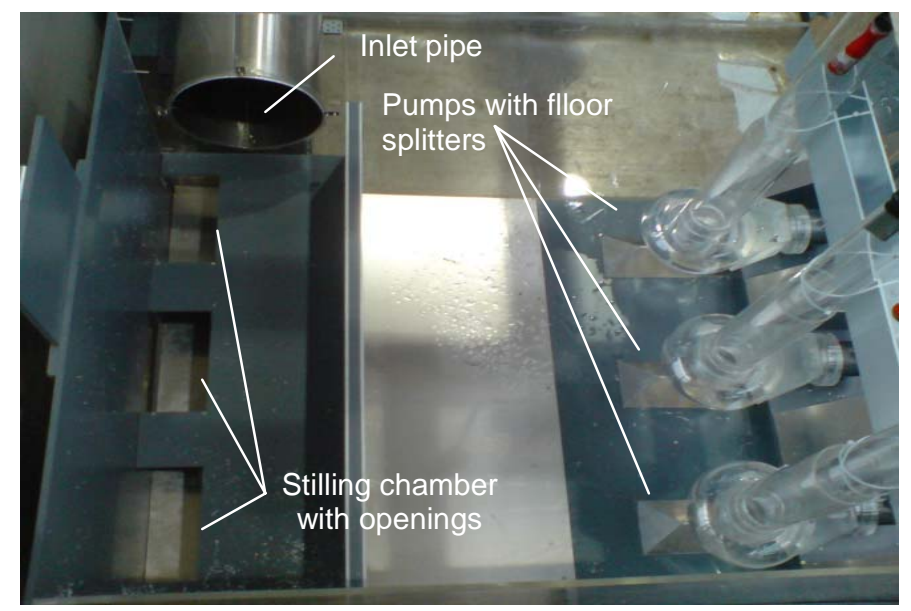

Fig. 5 Modeled waste water pumping station
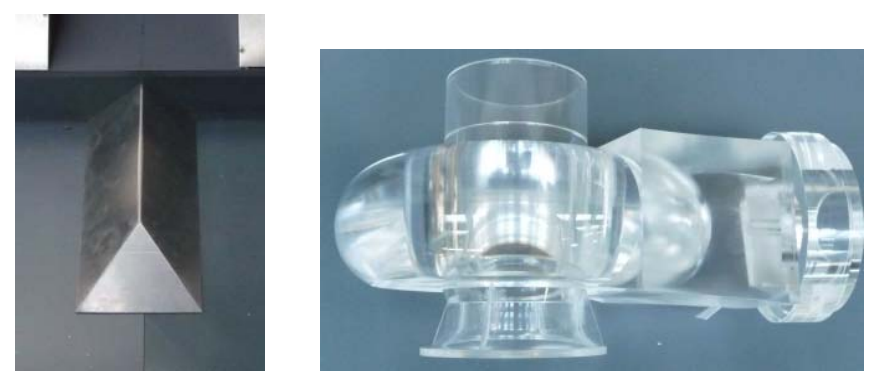

Fig. 6 Modeled $90^{\circ}$-floor-splitter and submersible motor pump

The inflow enters the stilling chamber and gets reflected at the top end. The slowed down flow can leave the stilling chamber through the openings at ground level and the side wall. Splitting up the flow into two different streams leads to a smooth flow deflection. The flow leaving the openings hits the guiding walls underneath the chamber and gets deflected towards the sump. The flow leaving over the side wall has an direct influence on the surface flow and in this way on the development of free surface vortex systems. Also the formation of possible floating sludge can be affected by surface-near flow. The strength of the influence depends on the water level inside the structure. 


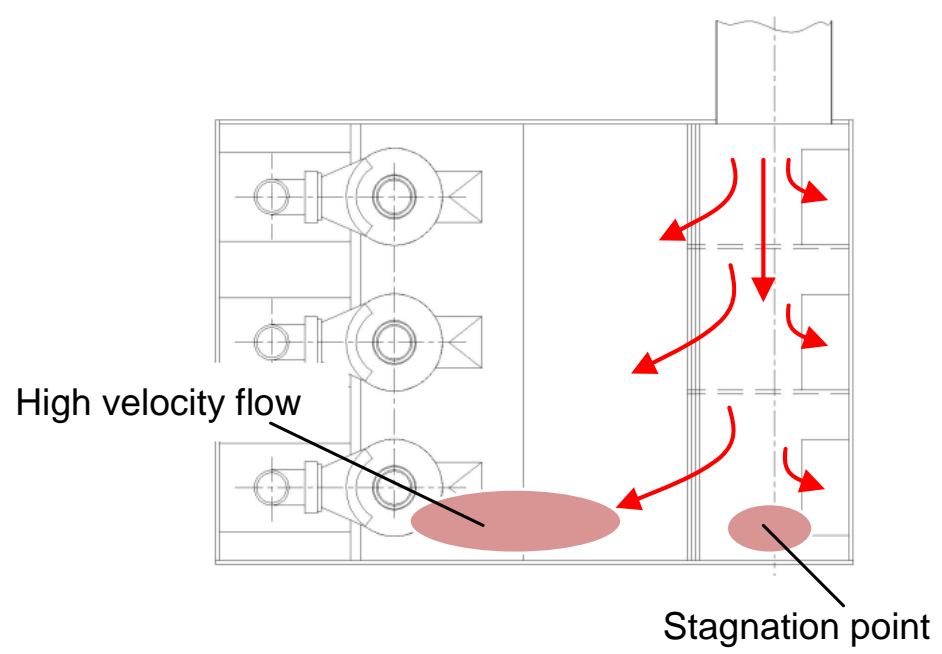

Fig. 7 Scheme of flow distribution (top view)

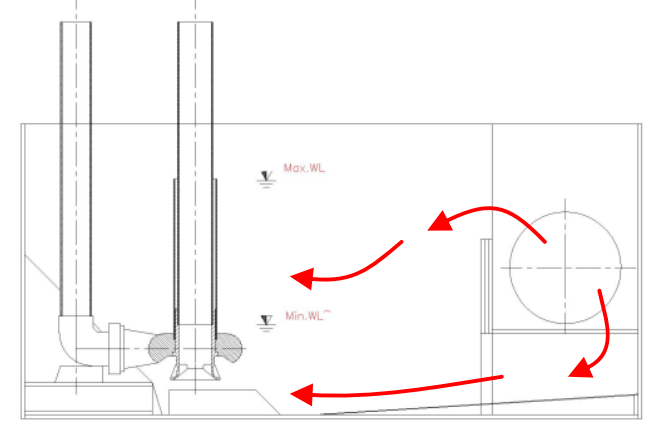

Fig. 8 Scheme of flow distribution (side view)

Concerning vortex formations and air entrainment the flow inside the structure can be sectioned into three phases, all depending on the water level.

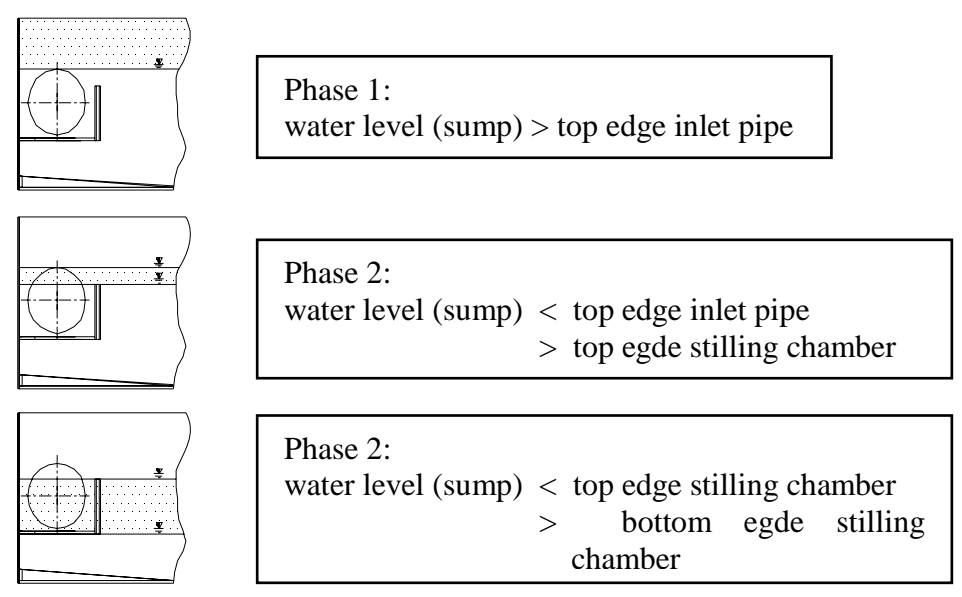

Fig. 9 Scheme of different flow conditions

With a water level above the top edge of the inlet pipe (Phase 1) calm free surface conditions could be detected. The low velocity surface flow only gets disturbed by the riser pipes of the pumps. These surface flow conditions imply the risk of evolving floating sludge in the prototype structure, as light solid parts and heavy foam formations grow to a solid layer on the surface. Another threat in this phase is the occurrence of free surface vortices. Due to the smooth surface vortex formations can evolve unobstructed. The potential for such a case increases with a descending water level and growing flow rates.

If the water decreases under the elevation of the inlet pipe top edge (Phase 2), flow distribution changes rapidly inside the structure. The inflow leaving the pipe partly hits the free surface, as well as the flow leaving the stilling chamber over the side wall. In this way, air gets entrained into the water. Depending on the flow rate and the water level, the air entrainment can cause foam formations. In this phase, no free surface problems are to be expected due to a constant surface turbulence. No air bubbles get transported from the stilling chamber to the pumps.

With a decreasing water level the free surface turbulence inside the stilling chamber as well as in the rest of the structure 
increases. When the water level is lower than the top edge of the stilling chamber side wall (Phase 3), the whole flow leaves the chamber through the floor openings. This leads immediately to higher velocities and with that to a more critical transport of air bubbles. Additionally the height of fall of the inlet flow - from the inlet pipe into the stilling chamber - increases with a reducing water level with the result of heavy air entrainment and foam formation. The problem in this operating period is air reaching the inlet nozzles of the pumps, leading to insufficient operating conditions, which can additionally harm all downstream processes. The design of the investigated structure was improved in previous model tests to avoid such flow conditions not in compliance with state of the art criteria. Most of the air ascends inside the stilling chamber. The air bubbles that leave through the openings ascend before reaching the pumps.

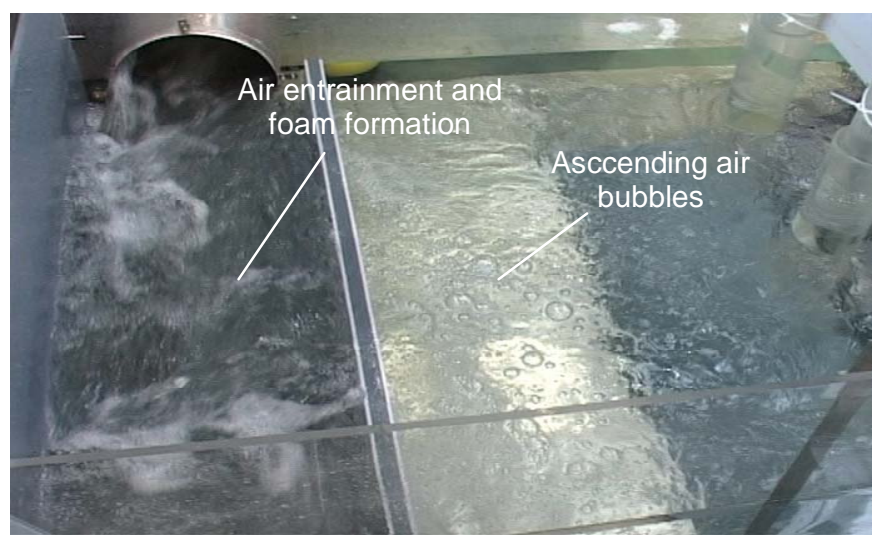

Fig. 10 Flow conditions inside model structure (phase 2)

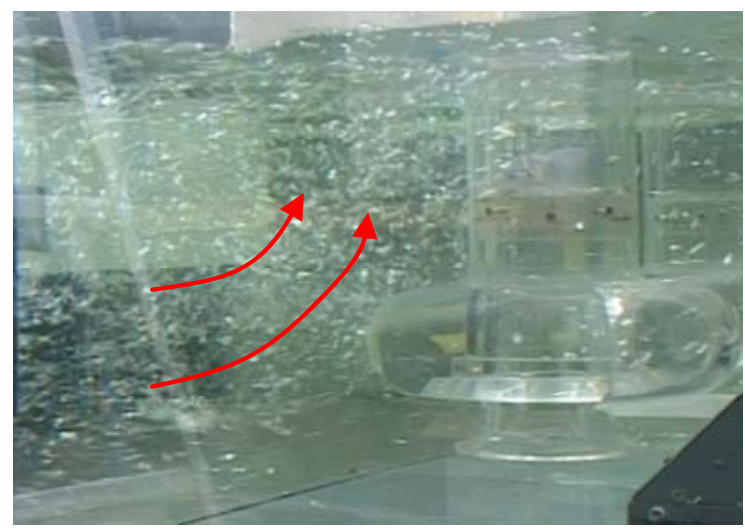

Fig. 11 Ascending air bubbles (phase 2)

Flow conditions become critical as the water level decreases lower than the elevation of the chamber openings. Strongly increasing air entrainment and foam formations can be detected. Due to increasing flow velocities air bubbles get transported into the direct suction area of the pumps. This leads to non-acceptable operating conditions. To adjust sufficient flow conditions, flow velocities have to be reduced by decreasing the flow rate. Based on the results of the described investigation, single pump operating is recommended when the water level is lower than the ground level of the stilling chamber. In this case the chamber does not work as a deflecting and calming component but as a mixer with the result of heavy air entrainment and foam formation into the pump sump.

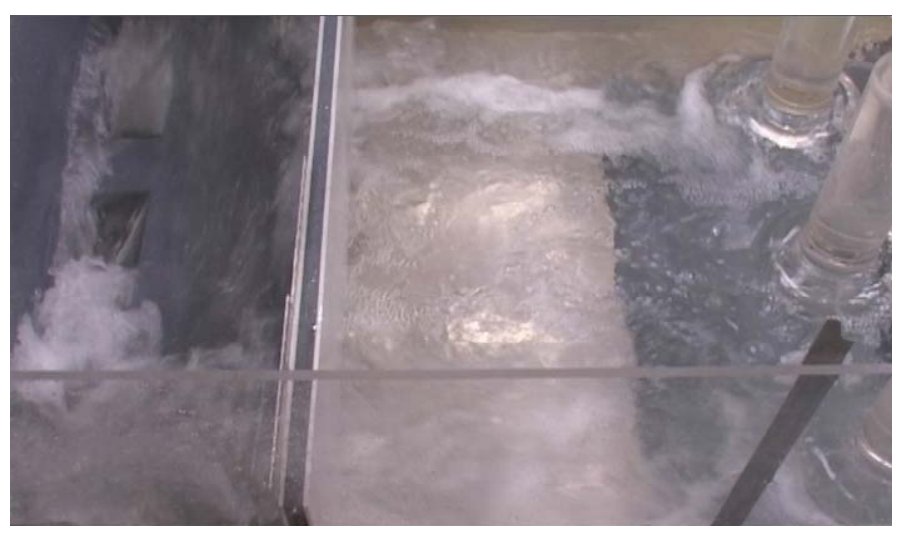

Fig. 12 High air entrainment and foam formation (phase 1) 
As described above, flow velocities in the area of the top end of the stilling chamber are higher than in the rest of the structure. Therefore the pump located near to this region bears the highest risk of getting charged by entrained air bubbles. Additionally, the velocity distribution has a major influence on single pump operating conditions. Due to a reduced flow rate, when operating only one pump, the open surface is calm, which are good starting conditions for vortex systems. The high velocity flow gets accelerated between the structure side wall and the pump casing. This initializes a rotational system, which increases to a free surface vortex of type 5-6 for all adjusted water levels.

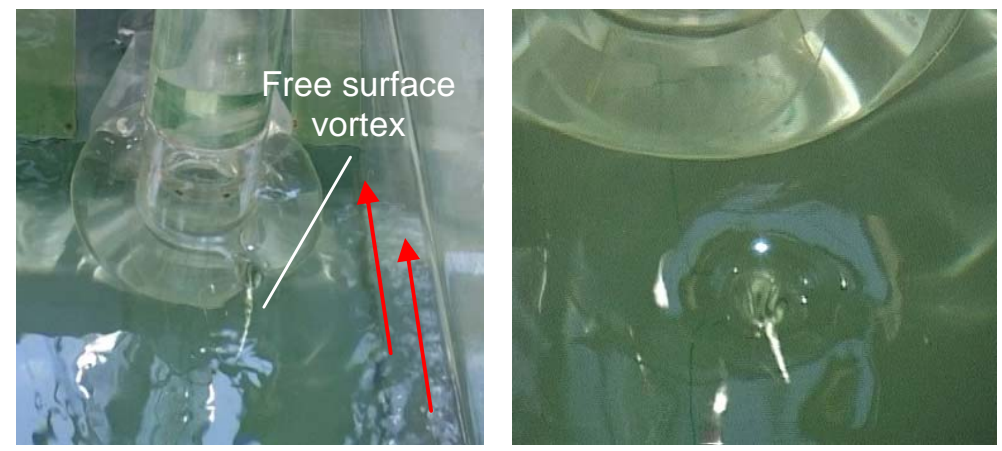

Fig. 13 Free surface vortex (Type 5-6)

Based on the test results, single pump operation cannot be recommended for pumps installed in the high velocity area next to the structures' side wall.

\section{Conclusion}

Evaluation, optimization and documentation of flow conditions for waste water pumping stations should always be done by physical model investigations. Compared with cooling water systems investigations for sewage water have to consider additional phenomena, such as floating sludge and heavy sedimentation. Additionally, waste water pumping stations normally are charged more unsteady. Due to the demand for smaller structures with increasing flow rates the specified limits of common standards sometimes are exceeded. All this leads to a different view on the investigation procedure. With physical model tests low velocity areas can be detected and optimized with flow guiding installations. Also the influence of entrained air, caused by increased heights of fall and reduced settling sections, can be estimated and optimized. Additionally, all state of the art evaluation criteria (e.g., evaluation of occurring vortex formation, velocity distributions and pre-rotation) are specified for waste water pumping stations. In this work, flow conditions for a compact waste water pumping station were evaluated with physical model investigations. In a first step flow conditions could be sectioned into three groups, depending on the water level. Each group was evaluated separately. It could be shown, that at higher water level free surface problems, such as free surface vortices and floating sludge, are more probable. With an decreasing water level air entrainment and foam formation strongly increase. Air, entrained in a stilling chamber, ascended within the investigated structure before reaching the pumps, as the water level did not fall below specified limits. For various flow rates these minimum water levels could be determined. For single pump operation, it could be specified that only pumps not located at the side walls should be used for operation. Pumps installed at side walls bear a higher risk of initializing free surface vortex formations, even at high water levels. Recommendations based on model test results lead to optimized operation conditions of prototype structures. For pumping stations with unsteady and critical boundary conditions, physical model investigations are the best tool of prediction.

\section{Nomenclature}

$\begin{array}{ll}C & \text { floor clearance [m] } \\ C & \text { flow velocity [m/s] } \\ D & \text { pump bell diameter [m] } \\ F_{r} & \text { Froude number } \\ L & \text { characteristic length [m] } \\ l & \text { characteristic length [m] } \\ R e & \text { Reynolds number }\end{array}$

$\begin{array}{ll}\mathrm{Re}_{\text {crit }} & \text { critical Reynolds number } \\ \mathrm{S} & \text { submergence [m] } \\ \mathrm{S} & \text { characteristic length [m] } \\ W & \text { bay width [m] } \\ W e & \text { Weber number } \\ X & \text { back wall clearance }[\mathrm{m}]\end{array}$

\section{References}

[1] Schäfer, F., 2008, "Untersuchung des Einflusses hydraulischer und mechanischer Anregungen auf das Betriebsverhalten einer axialen Rohrgehäusepumpe,” Kaiserslautern.

[2] GE. Hecker 1987, "Fundamentals of vortex intake flow," Swirling flow problems at intakes, IAHR, Hydraulic Structures design manual.

[3] Hydraulic Institute 1998, “American National Standard for Pump Intake Design,” ANSI/HI 9.8. 